\title{
A ictiofauna do Parque Estadual do Cantão, Estado do Tocantins, Brasil
}

\author{
Efrem Ferreira ${ }^{1,2}$, Jansen Zuanon ${ }^{1}$, Geraldo dos Santos $^{1} \&$ Sidinéia Amadio ${ }^{1}$ \\ ${ }^{1}$ Coordenação de Pesquisas em Biologia Aquática - CPBA, Instituto Nacional de Pesquisas da Amazônia- \\ INPA, Av. André Araujo, 2936 - CP 478, CEP 69011-970, Manaus - AM, Brasil \\ ${ }^{2}$ Autor para correspondência: Efrem Ferreira, e-mail: efrem@inpa.gov.br
}

FERREIRA, E., ZUANON, J., SANTOS, G. \& AMADIO, S. The fish fauna of the Parque Estadual do Cantão, Araguaia River, State of Tocantins, Brazil. Biota Neotrop. 11(2): http://www.biotaneotropica.org.br/v11n2/ en/inventory?article+bn01711022011

\begin{abstract}
The Parque Estadual do Cantão is a protected area in the Araguaia River. In a survey carried out during a complete hydrological cycle, 271 species of fish, belonging to 183 genera, 41 families and 12 orders were captured. Characidae was the most diverse family with 89 species (32.6\% of the total), followed by Loricariidae with 23 species (8.4\%) and Cichlidae with 21 species (7.7\%), ten families were represented by only one species. The results showed that the PE Cantão is an area with high diversity of fish species, and that the assemblies are still well preserved, so the existence and maintenance of this Park is of high interest for the preservation of the Araguaia River fish fauna.

Keywords: biodiversity, amazon, protected area, fisheries, CPUE.
\end{abstract}

FERREIRA, E., ZUANON, J., SANTOS, G. \& AMADIO, S. A ictiofauna do Parque Estadual do Cantão, Estado do Tocantins, Brasil. Biota Neotrop. 11(2): http://www.biotaneotropica.org.br/v11n2/pt/ inventory?article+bn01711022011

Resumo: O Parque Estadual do Cantão é uma área protegida no rio Araguaia. Em um inventário realizado durante um ciclo hidrológico completo, foram capturadas 271 espécies de peixes, pertencentes a 183 gêneros, 41 famílias e 12 ordens. Characidae foi a família mais diversa com 89 espécies (32,6\% do total), seguida por Loricariidae com 23 espécies $(8,4 \%)$ e Cichlidae com 21 espécies (7,7\%), dez famílias foram representadas por uma única espécie. Os resultados mostraram que o PE Cantão é uma área com alta diversidade de espécies de peixes, e que as assembleias ainda estão bem preservadas, de modo que a existência e manutenção deste Parque é de alto interesse para a preservação da fauna de peixes do rio Araguaia.

Palavras-chave: biodiversidade, amazônia, unidade de conservação, pesca, CPUE. 


\section{Introdução}

Este estudo foi realizado para inventariar a fauna de peixes do Parque Estadual do Cantão (PE Cantão), uma área protegida criada pelo governo do Estado do Tocantins no rio Araguaia (Figura 1). O parque tem $72 \mathrm{~km}$ de comprimento por 12 de largura, ocupando uma área de aproximadamente $890 \mathrm{~km}^{2}$, no curso médio do rio Araguaia, próximo da fronteira com o estado do Pará, entre as coordenadas $9^{\circ}$ $10^{\prime}$ S e 50 $10^{\prime}$ O. É uma importante zona de transição entre a floresta amazônica e o cerrado.
O PE Cantão forma uma grande planície aluvial, composta principalmente por areias de quartzo e sedimentos depositados pelos rios Javaés e Araguaia. A planície é formada principalmente por uma floresta pluvial, pertencente ao domínio amazônico, e tem cerca de 840 lagos, $150 \mathrm{~km}$ de meandros e canais naturais, assim como também por um tipo de cerrado localmente conhecido como "varjão", que é periodicamente inundado. As águas são claras como no rio Araguaia, com transparência medindo entre 0,4 e 2,0 m. O pH varia entre 5,2 e 7,9 e a condutividade elétrica entre 15,1 e $34,5 \mu \mathrm{S} / \mathrm{cm}$. Durante o

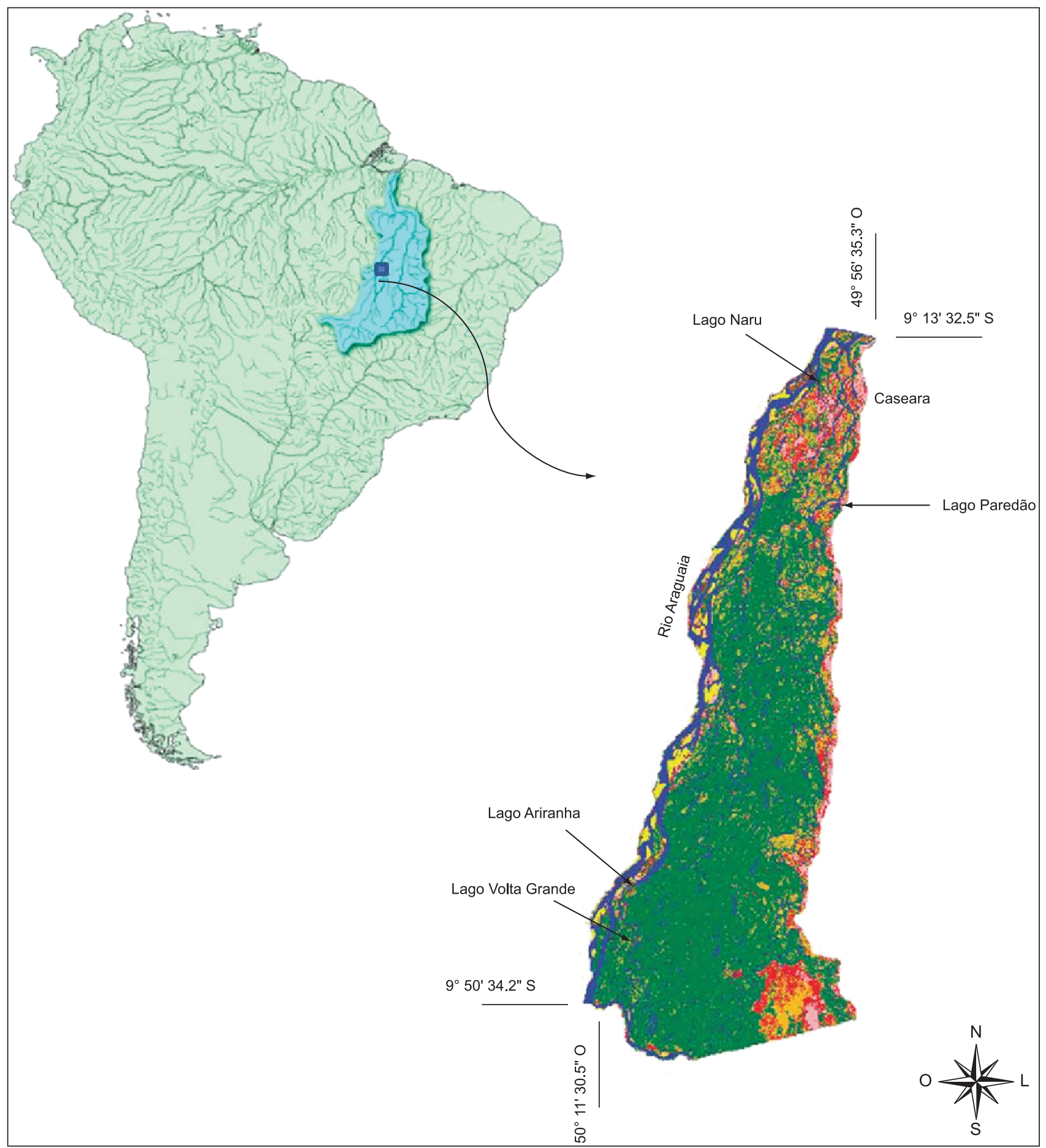

Figura 1. Parque Estadual do Cantão, mostrando os lagos amostrados (mapa da América do Sul de Goulding et al. 2003).

Figure 1. Parque Estadual do Cantão, showing the lakes sampled (South America map from Goulding et al., 2003). 
período de seca a maior parte dos canais secam ou são interrompidos por bancos de areia, e os lagos permanecem isolados. A área é relativamente bem preservada, com somente $8 \%$ de desmatamento, causado pelas queimadas para plantio agrícola.

Os arredores do parque são constituídos principalmente por cerrado, com predominância de savana aberta e por florestas de galeria. A vegetação, contudo, tem mudado em virtude do intensivo uso do solo, espacialmente para agricultura e pecuária de gado.

\section{Material e Métodos}

Quatro expedições de coleta foram realizadas, cobrindo os 4 períodos hidrológicos. Os peixes foram capturados com o auxílio de redes de emalhar (malhadeiras), rapichés, tarrafas e redes de cerco. Quatro lagos foram escolhidos para as amostragens durante um ano completo (Tabela 1). Além dos quatro lagos, vários outros ambientes, como praias e vegetação aquática flutuante, foram incluídos no programa de coletas.

Uma bateria de 10 malhadeiras com $10 \mathrm{~m}$ de comprimento, e altura ao redor de $2,5 \mathrm{~m}$, com tamanho de malha variando entre 24 e $120 \mathrm{~mm}$, entre nós opostos, foi usada em pescarias de 24 horas em cada um dos quatro lagos. Nas praias e vegetação flutuante foi utilizada uma rede de cerco com $15 \mathrm{~m}$ de comprimento, $3 \mathrm{~m}$ de altura e malha de $5 \mathrm{~mm}$. Todos os exemplares foram capturados e amostras de cada espécie foram fixadas em formol 10\% e preservadas em álcool 70\%. Após a identificação estes exemplares foram depositados na Coleção de Peixes do Instituto Nacional de Pesquisas da Amazônia (INPA), em Manaus, estado do Amazonas, Brasil.

\section{Resultados e Discussão}

Foram capturadas 271 espécies de peixes, pertencentes a 183 gêneros, 41 famílias e 12 ordens (Tabela 2). A proporção de espécies é similar àquela proposta por Roberts (1972) com a predominância de Ostariophysi: Characiformes (52,4\%), Siluriformes (26,9\%) e Gymnotiformes (5,2\%), seguido por Perciformes (9,6\%). As oito outras ordens foram responsáveis pelos 5,8\% restantes (Tabela 3).

Characidae foi a família mais diversa com 87 espécies $(32,1 \%$ do total), seguida por Loricariidae com 23 espécies (8,5\%), Cichlidae com 21 espécies (7,7\%), Anostomidae e Curimatidae com 14 espécies $(5,2 \%)$ cada, Doradidae com 12 espécies $(4,4 \%)$ e Pimelodidae com 11 espécies $(4,1 \%)$. Dez famílias foram representadas por somente uma espécie.

O número de espécies capturadas nesta pesquisa (271) indica uma diversidade muito alta, comparável a outros rios na Amazônia (Tabela 4), especialmente se considerando que este é apenas um pequeno trecho do rio Araguaia, e provavelmente o número de espécies aumentará à medida que os exemplares coletados sejam objeto de estudos mais detalhados.

As malhadeiras capturaram 129 espécies pertencentes a 94 gêneros, 24 famílias e oito ordens. O total estimado de espécies pelo Jackknife (Past software 2.03), (Hammer et al. 2001) variou entre 147 e 151, de modo que coletamos de 85 a $88 \%$ das espécies de peixes presentes neste trecho de rio (Tabela 5). A tabela 6 mostra as

Tabela 1. Coordenadas geográficas dos lagos amostrados no PE Cantão. Table 1. Geographical coordinates of the lakes sampled in the PE Cantão.

\begin{tabular}{|c|c|}
\hline Locais Amostrados & Coordenadas \\
\hline Lago Naru & $9^{\circ} 16^{\prime} 50,5^{\prime}$ S, 4959' 44,9” O \\
\hline Lago Paredão & $9^{\circ} 22^{\prime} 39,6^{\prime \prime} \mathrm{S}, 49^{\circ} 58^{\prime} 31,6^{\prime \prime} \mathrm{O}$ \\
\hline Lago Ariranhas & $9^{\circ} 43^{\prime} 47,5^{\prime \prime} \mathrm{S}, 50^{\circ} 09^{\prime} 14,5^{\prime \prime} \mathrm{O}$ \\
\hline Lago Volta Grande & $9^{\circ} 47^{\prime} 18,9^{\prime \prime} \mathrm{S}, 50^{\circ} 09^{\prime} 52,6^{\prime \prime} \mathrm{O}$ \\
\hline
\end{tabular}

Tabela 2. Lista das espécies de peixes capturadas no Parque Estadual do Cantão. *Espécies capturadas com malhadeiras em pescarias experimentais.

Table 2. List of fish species collected in Parque Estadual do Cantão. *Species caught with gillnets in experimental fisheries.

\begin{tabular}{|c|}
\hline Lista das espécies de peixes \\
\hline Rajiformes \\
\hline Potamotrygonidae \\
\hline Paratrygon aiereba (Müller \& Henle, 1841) \\
\hline \multirow{2}{*}{$\begin{array}{l}\text { Potamotrygon cf. motoro (Müller \& Henle, 1841)* } \\
\text { Osteoglossiformes }\end{array}$} \\
\hline \\
\hline Araipamidae \\
\hline Arapaima gigas (Schinz, 1822)* \\
\hline Osteoglossidae \\
\hline Osteoglossum bicirrhosum (Cuvier, 1829)* \\
\hline Clupeiformes \\
\hline Engraulidae \\
\hline Anchoviella carrikeri Fowler, 1940 \\
\hline Lycengraulis batesii (Günther, 1868)* \\
\hline Pristigasteridae \\
\hline Pellona castelnaeana (Valenciennes, 1847)* \\
\hline Pristigaster cayanna Cuvier, 1829* \\
\hline Characiformes \\
\hline Parodontidae \\
\hline Apareiodon machrisi Pavanelli \& Britski, 2003 \\
\hline Curimatidae \\
\hline Curimata acutirostris Vari \& Reis, 1995* \\
\hline Curimata inornata Vari, 1989* \\
\hline Curimatella dorsalis (Eigenmann \& Eigenmann, 1889)* \\
\hline Curimatella immaculata (Fernández-Yépez, 1948)* \\
\hline Curimatopsis crypticus Vari, 1982 \\
\hline Curimatopsis macrolepis (Steindachner, 1876) \\
\hline Cyphocharax gouldingi Vari, 1992* \\
\hline Cyphocharax leucostictus (Eigenmann \& Eigenmann, 1889) \\
\hline Cyphocharax notatus (Steindachner, 1908)* \\
\hline Cyphocharax plumbeus (Eigenmann \& Eigenmann, 1889)* \\
\hline Cyphocharax stilbolepis Vari, 1992* \\
\hline Psectrogaster amazonica Eigenmann \& Eigenmann, 1889* \\
\hline Steindachnerina gracilis Vari \& Vari, 1989* \\
\hline Prochilodontidae \\
\hline Prochilodus nigricans Agassiz, 1829* \\
\hline Semaprochilodus brama (Valenciennes, 1850)* \\
\hline Anostomidae \\
\hline Anostomoides laticeps (Eigenmann, 1912)* \\
\hline Anostomus anostomus (Linnaeus, 1758) \\
\hline Laemolyta fernandezi Myers, 1950* \\
\hline Laemolyta taeniata (Kner, 1859)* \\
\hline Leporellus vittatus (Velenciennes, 1850) \\
\hline Leporinus affinis Günther, 1864* \\
\hline Leporinus geminis Garavello \& Santos, 2009* \\
\hline Leporinus cylindriformis Borodin, 1929 \\
\hline Leporinus desmotes Fowler, 1914 \\
\hline Leporinus friderici (Bloch, 1794)* \\
\hline Leporinus julii Santos, Jégu \& Lima, 1996 \\
\hline Leporinus trifasciatus Steindachner, 1876* \\
\hline Leporinus unitaeniatus Garavello \& Santos, 2009* \\
\hline Schizodon vittatus (Valenciennes, 1850)* \\
\hline
\end{tabular}


Tabela 2. Continuação.

\begin{tabular}{|c|}
\hline Lista das espécies de peixes \\
\hline Chilodontidae \\
\hline Caenotropus labyrinthicus (Kner, 1858)* \\
\hline Chilodus punctatus Müller \& Troschel, 1844 \\
\hline Crenuchidae \\
\hline Characidium sp. (claro) \\
\hline Characidium sp. (escuro) \\
\hline Melanocharacidium cf. auroradiatum Costa \& Vicente, 1994 \\
\hline Hemiodontidae \\
\hline Anodus orinocensis (Steindachner, 1887)* \\
\hline Argonectes robertsi Langeani, 1999* \\
\hline Bivibranchia fowleri (Steindachner, 1908) \\
\hline Bivibranchia velox (Eigenmann \& Myers, 1927) \\
\hline Hemiodus goeldii Steindachner, 1908* \\
\hline Hemiodus microlepis Kner, 1858* \\
\hline Hemiodus unimaculatus (Bloch, 1794)* \\
\hline Characidae \\
\hline Agoniates halecinus Müller \& Troschel, $1845^{*}$ \\
\hline Aphyocharax alburnus (Günther, 1869) \\
\hline Aphyocharax avary Fowler, 1913 \\
\hline Astyanax sp.* \\
\hline Astyanax sp. "J" \\
\hline Brycon falcatus Müller \& Troschel, 1844 \\
\hline Brycon gouldingi Lima, 2004* \\
\hline Brycon aff. pesu Müller \& Troschel, 1845 \\
\hline Brycon cf. pesu Müller \& Troschel, $1845^{*}$ \\
\hline Bryconops aff. affinis (Günther, 1864)* \\
\hline Bryconops alburnoides Kner, 1858* \\
\hline Bryconops cf. collettei Chernoff \& Machado-Allison, 2005 \\
\hline Bryconops sp. $*$ \\
\hline Catoprion mento (Cuvier, 1819)* \\
\hline Chalceus epakros Zanata \& Toledo-Piza, 2004* \\
\hline Charax sp.* \\
\hline Charax sp. 2 \\
\hline Clupeacharax anchoveoides Pearson, 1924 \\
\hline Creagrutus cracentis Vari \& Harold, 2001 \\
\hline Creagrutus menezesi Vari \& Harold, 2001 \\
\hline Exodon paradoxus Muller \& Troschel, 1844* \\
\hline Gymnocorymbus thayeri Eigenmann, 1908* \\
\hline Hemigrammus aff. levis Durbin, 1908 \\
\hline Hemigrammus stictus (Durbin, 1909) \\
\hline Hemigrammus aff. ocellifer (Steindachner, 1882) \\
\hline Hemigrammus levis Durbin, 1908 \\
\hline Hemigrammus ocellifer (Steindachner, 1882) \\
\hline Hemigrammus sp. 2 \\
\hline Hemigrammus sp. 3 \\
\hline Hyphessobrycon sp. \\
\hline Hyphessobrycon sp. 3 \\
\hline Iguanodectes cf. spilurus (Günther, 1864) \\
\hline Jupiaba acanthogaster (Eigenmann, 1911) \\
\hline Jupiaba cf. essequibensis (Eigenmann, 1909) \\
\hline Jupiaba polylepis (Günther, 1864) \\
\hline Knodus heteresthes (Eigenmann, 1908) \\
\hline Knodus sp. "sem mancha" \\
\hline
\end{tabular}

Lista das espécies de peixes

Metynnis hypsauchen (Müller \& Troschel, 1844)*

Metynnis lippincottianus (Cope, 1870)*

Metynnis maculatus (Kner, 1858) *

Microschemobrycon geisleri Géry, 1973

Microschemobrycon sp. 2

Moenkhausia aff. chrysargyrea (Günther, 1864)*

Moenkhausia aff. collettii (Steindachner, 1882)

Moenkhausia aff. lepidura (Kner, 1858)

Moenkhausia aff. newtoni Travassos, 1964

Moenkhausia cf. ceros Eigenmann, 1908

Moenkhausia cf. lepidura (Kner, 1858)

Moenkhausia cf. oligolepis (Günther, 1864)

Moenkhausia collettii (Steindachner, 1882)

Moenkhausia dichroura (Kner, 1858)*

Moenkhausia gr. lepidura (Kner, 1858)

Moenkhausia gracilima (Eigenmann, 1908)

Moenkhausia grandisquamis (Müller \& Troschel, 1845)

Moenkhausia jamesi Eigenmann, 1908

Moenkhausia lepidura (Kner, 1858)

Moenkhausia megalops (Eigenmann, 1907)

Moenkhausia oligolepis (Günther, 1864)

Moenkhausia pyrophthalma Costa, 1994

Moenkhausia sp. "alta"*

Moenkhausia sp. * $^{*}$

Moenkhausia sp.2

Myleus setiger Müller \& Troschel, 1844*

Myloplus arnoldi Ahl, 1936

Myloplus rubripinnis (Müller \& Troschel, 1844)*

Myloplus schomburgki (Jardine \& Schomburgk, 1841)*

Myloplus torquatus (Kner, 1858)*

Mylossoma duriventre (Cuvier, 1818)*

Odontostilbe sp.

Parapristella georgiae Géry, 1964

Phenacogaster cf. calverti (Fowler, 1941)

Piaractus brachypomus (Cuvier, 1818)*

Poptella compressa (Günther, 1864)*

Pristobrycon eigenmanni Norman, 1929*

Pygocentrus nattereri Kner, 1858*

Roeboexodon geryi Myers, 1960

Roeboides affinis (Günther, 1868)*

Serrapinnus sp.

Serrasalmus geryi Jégu \& Santos, 1988*

Serrasalmus gibbus Castelnau, 1855*

Serrasalmus maculatus Kner, 1858*

Serrasalmus rhombeus (Linnaeus, 1766)*

Tetragonopterus chalceus Spix \& Agassiz, 1829*

Thayeria boehlkei Weitzman, 1957

Tometes sp.

Triportheus albus Cope, 1872*

Triportheus auritus (Valenciennes, 1850)*

Triportheus trifurcatus (Castelnau, 1855)*

Acestrorhynchidae

Acestrorhynchus falcirostris (Cuvier, 1819)*

Acestrorhynchus microlepis (Schomburgk, 1841)* 
Tabela 2. Continuação.

\begin{tabular}{|c|}
\hline Lista das espécies de peixes \\
\hline Cynodontidae \\
\hline Cynodon gibbus Spix \& Agassiz, 1829* \\
\hline Hydrolycus armatus (Jardine \& Schomburgk, 1841)* \\
\hline $\begin{array}{l}\text { Hydrolycus tatauaia Toledo-Pizza, Menezes \& Santos, } \\
\text { 1999* }\end{array}$ \\
\hline Rhaphiodon vulpinus Spix \& Agassiz, 1829* \\
\hline Erythrinidae \\
\hline Erythrinus erythrinus (Bloch \& Schneider, 1801) \\
\hline Hoplias malabaricus (Bloch, 1794)* \\
\hline Lebiasinidae \\
\hline Copella sp. \\
\hline Pyrrhulina aff. brevis Steindachner, 1876 \\
\hline Ctenolucidae \\
\hline Boulengerella cuvieri (Agassiz, 1829)* \\
\hline Boulengerella maculata (Valenciennes, 1850)* \\
\hline Siluriformes \\
\hline Cetopsidae \\
\hline Cetopsis coecutiens(Lichtenstein, 1819) \\
\hline Aspredinidae \\
\hline Bunocephalus coracoideus (Cope, 1874) \\
\hline Trichomycteridae \\
\hline Haemomaster venezuelae Myers, 1927 \\
\hline Henonemus punctatus (Boulenger, 1887) \\
\hline Ituglanis cf. amazonicus (Syeincachner, 1882) \\
\hline Ochmacanthus sp. \\
\hline Pseudostegophilus sp. \\
\hline Stegophilus sp. 1 \\
\hline Vandellia sp. \\
\hline Callichthyidae \\
\hline Corydoras aff. xinguensis Nijssen, 1972 \\
\hline Hoplosternum litoralle (Hancock, 1828) \\
\hline Megalechis picta (Müller \& Troschel, 1848) \\
\hline Loricariidae \\
\hline Ancistrus sp.* \\
\hline Baryancistrus niveatus (Castelnau, 1855) \\
\hline Dekeyseria amazonica Rapp Py-Daniel, 1985* \\
\hline Farlowella amazona (Günther, 1864) \\
\hline Hemiodontichthys acipenserinus (Kner, 1853) \\
\hline Hypoptopoma gulare Cope, $1878^{*}$ \\
\hline Hypostomus cf. carinatus (Steindachner, 1881)* \\
\hline Hypostomus sp.* \\
\hline Hypostomus sp. $3 *$ \\
\hline Leporacanthicus galaxias Isbrücker \& Nijssen, 1989 \\
\hline Loricaria sp. \\
\hline Loricariichthys acutus (Valenciennes, 1836)* \\
\hline Panaque aff. nigrolineatus (Peters, 1877) \\
\hline Parancistrus aurantiacus (Castelnau, 1855) \\
\hline Peckoltia aff. oligospila (Günther, 1864) \\
\hline Peckoltia aff. vittata (Steindachner, 1881) \\
\hline Peckoltia sp. (pinta branca) \\
\hline Pseudoloricaria laeviuscula (Valenciennes, 1840) \\
\hline Pseudoloricaria sp. \\
\hline Pterygoplichthys joselimaianus Weber, 1991* \\
\hline
\end{tabular}

\section{Lista das espécies de peixes}

Rineloricaria sp. *

Squaliforma emarginata (Valenciennes, 1840)*

Sturisoma sp.*

Pseudopimelodidae

Microglanis sp.

Pseudopimelodus sp.

Heptapteridae

Cetopsorhamdia sp.

Imparfinis sp.

Pimelodella cf. gracilis (Valenciennes, 1835)

Pimelodella sp.*

\section{Pimelodidae}

Hemisorubim platyrhynchos (Valenciennes, 1840)*

Hypophthalmus marginatus Valenciennes, 1840*

Phractocephalus hemioliopterus (Bloch \& Schneider, 1801)*

Pimelodina flavipinnis Steindachner, 1877

Pimelodus cf. blochii Valenciennes, 1840*

Pimelodus ornatus Kner, 1858

Pimelodus tetramerus Ribeiro \& Lucena, 2006

Pinirampus pirinampu (Spix \& Agassiz, 1829)*

Pseudoplatystoma punctifer (Castelnau, 1855)*

Sorubim lima (Bloch \& Schneider, 1801)*

Sorubimichthys planiceps (Spix \& Agassiz, 1829)

\section{Doradidae}

Amblydoras affinis (Kner, 1855)

Anadoras sp.

Doras zuanoni Sabaj Pérez \& Birindelli, 2008*

Hassar orestis (Steindachner, 1875)*

Leptodoras cf. acipenserinus (Günther, 1868)

Leptodoras hasemani (Steindachner, 1915)

Nemadoras sp.

Nemadoras trimaculatus (Boulenger, 1898)*

Oxydoras niger (Valenciennes, 1821)*

Platydoras armatulus (Valenciennes, 1840)*

Pterodoras granulosus (Valenciennes, 1821)

Rhinodoras cf. dorbignyi (Kner, 1855)

Auchenipteridae

Ageneiosus cf. ucayalensis Castelnau, 1855*

Ageneiosus inermis (Linnaeus, 1766)*

Auchenipterichthys coracoideus (Eigenmann \& Allen, 1942)*

Auchenipterichthys longimanus (Günther, 1864)

Auchenipterus nuchalis (Spix \& Agassiz, 1829)*

Parauchenipterus galeatus (Linnaeus, 1758)*

Tatia sp. *

Tocantinsia piresi (Miranda Ribeiro, 1920)*

Trachycorystes $s p . *$

Gymnotiformes

Gymnotidae

Gymnotus carapo Linnaeus, 1758

\section{Sternopygidae}

Distocyclus cf. conirostris (Eigenmann \& Allen, 1942)

Eigenmannia aff. trilineata López \& Castello, 1966

Eigenmannia cf. virescens (Valenciennes, 1842) 
Tabela 2. Continuação.

\begin{tabular}{|c|}
\hline Lista das espécies de peixes \\
\hline Eigenmannia limbata (Schreiner \& Miranda Ribeiro, 1903)* \\
\hline Eigenmannia macrops (Boulenger, 1897) \\
\hline Sternopygus macrurus (Bloch \& Schneider, 1801) \\
\hline Sternopygus xingu Albert \& Fink, 1996* \\
\hline Rhamphichthyidae \\
\hline Gymnorhamphichthys petiti Géry \& Vu-Tân-Tuê, 1964 \\
\hline Rhamphichthys marmoratus Castelnau, 1855* \\
\hline Hypopomidae \\
\hline Brachyhypopomus brevirostris (Steindachner, 1868) \\
\hline Brachyhypopomus sp. "base da anal escura" \\
\hline Apteronotidae \\
\hline Apteronotus aff. albifrons (Linnaeus, 1766) \\
\hline Platyurosternarchus cf. macrostomus (Günther, 1870) \\
\hline Cyprinodontiformes \\
\hline Rivulidae \\
\hline Rivulus sp. \\
\hline Poeciliidae \\
\hline Pamphorichthys araguaiensis Costa, 1991 \\
\hline Beloniformes \\
\hline Belonidae \\
\hline Potamorrhaphis guianensis (Jardine, 1843) \\
\hline Pseudotylosurus microps (Günther, 1866) \\
\hline Synbranchiformes \\
\hline Synbranchidae \\
\hline Synbranchus sp. 1 \\
\hline Synbranchus sp. 2 \\
\hline Perciformes \\
\hline Sciaenidae \\
\hline Pachyurus junki Soares \& Casatti, 2000* \\
\hline Pachyurus paucirastrus Aguilera, 1983 \\
\hline Pachyurus schomburgkii Günther, 1860 \\
\hline
\end{tabular}

Tabela 3. Porcentagem do número de espécies de peixes de cada Ordem capturada por malhadeiras e por todos os outros aparelhos no Parque Estadual do Cantão.

Table 3. Percentage of the number of fish species of each Order captured by gillnets and by all fishing devices in the Parque Estadual do Cantão.

\begin{tabular}{lcc}
\hline \multicolumn{1}{c}{ Ordem } & Geral & Malhadeiras \\
\hline Characiformes & 52,4 & 56,5 \\
Siluriformes & 26,9 & 24,8 \\
Perciformes & 9,6 & 10,9 \\
Gymnotiformes & 5,2 & 2,3 \\
Clupeiformes & 1,5 & 2,3 \\
Rajiformes & 0,7 & 0,8 \\
Osteoglossiformes & 0,7 & 1,6 \\
Cyprinodontiformes & 0,7 & 0,0 \\
Beloniformes & 0,7 & 0,0 \\
Synbranchiformes & 0,7 & 0,0 \\
Pleuronectiformes & 0,4 & 0,8 \\
Tetraodontiformes & 0,4 & 0,0 \\
Número de Espécies & 271 & 129 \\
\hline
\end{tabular}

\section{Lista das espécies de peixes}

Petilipinnis grunniens (Jardine, 1843)*

Plagioscion squamosissimus (Heckel, 1840)*

\section{Cichlidae}

Apistogramma sp.

Astronotus cf. ocellatus (Agassiz, 1831)*

Biotodoma cupido (Heckel, 1840)*

Caquetaia spectabilis Steindachner, 1875

Chaetobranchus flavescens Heckel, 1840*

Cichla kelberi Kullander \& Ferreira, 2006*

Cichla piquiti Kullander \& Ferreira, 2006*

Cichlasoma araguaiense Kullander, 1983*

Crenicichla aff. wallacii Regan, 1905

Crenicichla inpa Ploeg, 1991

Crenicichla labrina (Spix \& Agassiz, 1831)*

Crenicichla regani Ploeg, 1989

Crenicihla lugubris Heckel, 1840*

Crenicihla reticulata (Heckel, 1840)

Geophagus aff. altifrons Heckel, 1840*

Hypselecara temporalis (Günther, 1862)

Laetacara araguaiae (Costa, 2009)

Mesonauta acora (Castelnau, 1855)*

Retroculus lapidifer (Castelnau, 1855)

Satanoperca jurupari (Heckel, 1840)

Satanoperca sp.*

Pleuronectiformes

Achiridae

Hypoclinemus mentalis (Günther, 1862)*

Tetraodontiformes

Tetraodontidae

Colomesus asellus (Müller \& Troschel, 1849)

Tabela 4. Número de espécies de peixes registradas para diferentes bacias hidrográficas no Brasil.

Table 4. Number of fish species registered for different basins in Brazil

\begin{tabular}{lcc}
\hline \multicolumn{1}{c}{ Rio } & N & Fonte \\
\hline Várzea do rio Amazonas & 647 & Zuanon et al. 2007 \\
Rio Branco & 527 & Ferreira et al. 2007 \\
Rio Negro & 450 & Goulding et al. 1988 \\
Rio Trombetas & 342 & Ferreira 1993 \\
Rio Jaú & 318 & J. Zuanon \& \\
& & E. Ferreira pers. obs. pess. \\
Cantão & $\mathbf{2 7 1}$ & Esta pesquisa \\
Pantanal & 269 & Britski et al. 2007 \\
Rio Tocantins & 217 & Santos et al. 2004 \\
Rio Mucajai & 126 & Ferreira et al. 1988 \\
\hline
\end{tabular}


características ecológicas desta assembleias baseadas nas capturas com malhadeiras estimadas pelo programa Past 2.03 (Hammer et al. 2001).

Ferreira et al. (1988) e Ferreira (1993) apresentam valores médios para o Índice de Diversidade de Shannon-Wiener para ambientes amazônicos que variam entre 2,18 para o rio Curuá-Uma e 6,18 para o rio Trombetas. Os valores estimados para o PE Cantão situam-se entre estes extremos.

Os valores de Equitabilidade foram considerados elevados, mesmo para padrões amazônicos, e similar àqueles encontrados para o rio Trombetas (Ferreira 1993)

Os resultados das pescarias experimentais com malhadeiras mostraram valores muito elevados de captura por unidade de esforço (CPUE), entre os maiores já registrados para ambientes aquáticos amazônicos (Tabela 7).

A única outra informação disponível para o rio Araguaia é de uma pesquisa realizada em um trecho a montante da ilha do Bananal (Tejerina-Garro et al. 1998), contudo os métodos utilizados foram

Tabela 5. Número total de espécies estimado pelo método JackKnife.

Table 5. Total number of species estimated by Jackknife.

\begin{tabular}{llll}
\hline Chao 2: & 140,521 & Chao 2 variance: & 1,04348 \\
Jackknife 1: & 147 & - & \\
Jackknife 2: & 151,333 & - \\
Bootstrap: & 138,113 & - \\
\hline
\end{tabular}

diferentes do que utilizamos em nosso estudo e os valores encontrados foram metade dos nossos. Também os valores para biomassa encontrados no PE Cantão são maiores que aquele encontrados para maioria de outros locais amostrados na Amazônia, com exceção do Lago do Inácio, um lago de várzea do rio Amazonas próximo a Manaus (Saint-Paul et al. 2000). Assim, o PE Cantão pode ser caracterizado como um local com alta produtividade biológica, especialmente considerando que este é um sistema de águas claras. Levando em consideração esta elevada produtividade e riqueza de espécies podemos também inferir que este ambiente deve estar em bom estado de conservação.

A fauna de peixes encontrada no rio Araguaia revela uma mistura de espécies da planície do rio Amazonas (Psectrogaster amazonica, Serrasalmus maculatus, Pygocentrus nattereri, Prochilodus nigricans, Mylossoma duriventre, Leporinus trifasciatus, Pterodoras granulosus, Hypselecara temporalis), e de alguns tributários do baixo rio Amazonas (Semaprochilodus brama, Retroculus lapidifer, Schizodon vittatus), com outras espécies típicas da bacia do rio Tocantins (Brycon gouldingi, Leporinus affinis, Serrasalmus gibbus, S. geryi, Triportheus trifurcatus, Curimata acutirostris, Steindachnerina gracilis, Cichla kelberi, Cichla piquiti, Cichlasoma araguaiense). Também espécies dos escudos do Brasil Central e das Guianas foram encontradas (Exodon paradoxus, Roeboexodon geryi, Bivibranchia velox, Baryancistrus niveatus, Laemolyta fernandezi, Leporellus vittatus, Leporinus julii, L. desmotes). Assim a ictiofauna do PE Cantão parece ser a soma de uma variedade de espécies de vários rios e sistemas, resultando em uma combinação peculiar com alta abundância e riqueza de espécies.

Tabela 6. Índices ecológicos calculados para as capturas com malhadeiras nos 4 lagos do PE Cantão.

Table 6. Ecological indexes calculated for the gillnets catches in four lakes of the PE Cantão.

\begin{tabular}{lcccc}
\hline & Ariranha & Naru & Paredão & Total \\
Riqueza S & 100 & 88 & 97 & 92 \\
Exemplares & 1828 & 2353 & 3522 & 1707 \\
Dominância D & 0,04927 & 0,1641 & 0,1002 & 0,05735 \\
Shannon H & 3,642 & 2,934 & 3,009 & 3,494 \\
Evenness & 0,9507 & 0,8359 & 0,8998 & 0,05192 \\
Equitabilidade & 0,3816 & 0,2136 & 0,2089 & 0,9427 \\
\hline
\end{tabular}

Tabela 7. Valores da Captura por Unidade de Esforço (CPUE) para pescarias experimentais com malhadeiras para alguns rios e lagos na região amazônica. - = não disponível

Table 7. Values of Capture per Unit of Effort (CPUE) for the experimental fisheries with gillnets for some rivers and lakes in Amazon region. - = not available

\begin{tabular}{|c|c|c|c|}
\hline \multirow[t]{2}{*}{ Local } & \multicolumn{2}{|c|}{ CPUE (m²/24 horas) } & \multirow[t]{2}{*}{ Fonte } \\
\hline & Biomassa (g) & Exemplares & \\
\hline Lago do Inácio & 190,00 & - & Saint-Paul et al. 2000 \\
\hline PE Cantão & 181,40 & 1,59 & Este artigo \\
\hline Rio Trombetas & 128,38 & 0,58 & Ferreira 1995 \\
\hline Rio Tocantins & 114,00 & - & Merona 1986/87 \\
\hline Rio Mucajaí & 89,65 & 0,43 & Ferreira et al. 1988 \\
\hline Rio Uatumã & 72,80 & - & Ferreira et al. 1988 \\
\hline Rio Jaú & 46,70 & 0,54 & J. Zuanon \& E. Ferreira obs. pessoal \\
\hline Lago do Prato & 41,00 & - & Saint-Paul et al. 2000 \\
\hline Rio Miuá (Rio Negro) & 26,88 & - & J. Zuanon \& E. Ferreira obs. pessoal \\
\hline
\end{tabular}




\section{Conclusão}

Nossos resultados mostraram que o PE Cantão é uma área com alta diversidade de espécies de peixes, e que as assembleias ainda estão bem preservadas, como indicado pelos valores de CPUE. A existência e manutenção deste Parque é de alto interesse para a preservação da ictiofauna do rio Araguaia.

\section{Agradecimentos}

Nós gostaríamos de agradecer à SEPLAN e a Naturatins órgãos do governo do Estado do Tocantins, e a Sra. Angélica Beatriz Gonçlaves pelo apoio durante as coletas.

\section{Referências Bibliográficas}

BRITSKI, H.A., SILIMON, K.Z. S. \& LOPES, B.S. 2007. Peixes do Pantanal - Manual de identificação. 2nd ed. Embrapa, Brasília, 227 p.

FERREIRA, E.J.G. 1993. Composição, distribuição e aspectos ecológicos da ictiofauna de um trecho do rio Trombetas, na área de influência da futura UHE Cachoeira Porteira, Estado do Pará, Brasil. Acta Amazon. 23(1-4; supl.):1-88.

FERREIRA, E.J.G., SANTOS, G.M. \& JEGU, M. 1988. Aspectos ecológicos da ictiofauna do rio Mucajaí, na área da ilha Paredão, Roraima, Brasil. Amazoniana 10(3):339-352.
FERREIRA, E., ZUANON, J., FORSBERG, B., GOULDING, M. \& BRIGLIA-FERREIRA, S.R. 2007. Rio Branco. Peixe, ecologia e conservação de Roraima. Amazon Conservation Association (ACA)/ Instituto Nacional de Pesquisas da Amazônia (INPA)/Sociedade Civil Mamirauá, Lima, 201 p.

GOULDING, M., CARVALHO, M.L. \& FERREIRA, E.G. 1988. Rio Negro: Rich Life in Poor Water. Amazonian Diversity and Food Chain Ecology as Seen through Fish Communities. SPB Academic Publishing, 200 p.

HAMMER, O., HARPER, D.A.T. \& RYAN, P.D. 2001. PAST: Paleontological Statistics software package for education and data analysis. Palaeontol. Electronica 4(11):1-9.

MERONA, B. 1987. Aspectos ecológicos da ictiofauna no baixo Tocantins. Acta Amazon., 16/17(n. único):109-124.

ROBERTS, T.R. 1972. Ecology of fishes in the Amazon and Congo Basins. Bull. Mus. Comp. Zool., 143(2):117-147.

SAINT-PAUL, U.,ZUANON, J., VILLACORTA CORREA, M.A., GARCIA, M., FABRÉ, N.N., BERGER, U. \& JUNK, W.J. 2000. Fish communities in central Amazonian white and blackwater floodplains. Env. Biol. Fishes, 57:235-250. http://dx.doi.org/10.1023/A:1007699130333

SANTOS, G. M., MERONA, B., JURAS, A.A. \& JÉGU, M. 2004. Peixes do Baixo Rio Tocantins: 20 anos depois da Usina Hidrelétrica Tucuruí. Eletronorte, Brasília, $216 \mathrm{p}$.

ZUANON, J., RAPP PY-DANIEL, L.H., FERREIRA, E.J.G., CLARO JUNIOR, L.H. \& MENDONÇA, F.P. 2007. Padrões de distribuição da ictiofauna na várzea do sistema Solimões-Amazonas, entre Tabatinga (AM) e Santana (AP). In Conservação da Várzea. Identificação e caracterização de regiões biogeográficas. (A.L. Albernaz, Org.). Ibama/ Provárzea. Manaus, 237-285.

Recebido em 22/10/2010

Versão reformulada recebida em 15/02/2011

Publicado em 06/05/2011 